\title{
Offshore towed hydrophone linear array: principle, application, and data acquisition results
}

\author{
Jin Chen ${ }^{1,2}$, Fa-jie Duan ${ }^{1 *}$, Jia-jia Jiang ${ }^{1}$, Yan-chao Li ${ }^{1}$ and Xiang-ning Hua ${ }^{1}$
}

\begin{abstract}
An underwater acoustic sensing array was presented in this article. With the high-precision sampling clock generation and transmission system, the array can acquire signals synchronously in sub-microsecond level, which is important in offshore environment. Meanwhile, real-time data transmission and storage system was established. All of the data received in host computer can be saved and displayed immediately. Data acquisition experiment was implemented in freshwater reservoir near Tianjin city,China, and the results of the signal wave show that the acquisition and transmission system of hydrophone array can be used to get the underwater information by acoustic exploration.
\end{abstract}

Keywords: Offshore acoustic exploration, Towed hydrophone linear array, Synchronous signal acquisition

\section{Introduction}

Acoustic exploration is one of the most important methods to acquire information from the ocean. Conventional wisdom holds that cabled ocean observatories are more widely used compared to wireless systems. While application fields of the wireless underwater sensor networks (WNSN) are relatively narrow. For example, VENUS (Victoria Experimental Network Under the Sea) and NEPTUNE (North-East Pacific Undersea Networked Experiments) ocean observatories are established in Canada, which are the two oceanic projects of University of Victoria in Canada [1]. VENUS is used in the coastal ocean, while NEPTUNE in the deep ocean [1]. But things have been changed in recent years. One application of WNSN was depicted in [2]. Deployment of networks can be cabled, fixed, and wireless. With the development of sensor technology and wireless communication technology, WNSN are no longer just in the secondary status nowadays. As another type of wireless sensing tool, autonomous wave glider was reported in [3], which is used for long-term working to conduct acoustic exploration. The power of glider is generated autonomously by waves. Glider and rudder are connected to a float by an umbilical. Compared to other vehicles, the self-noise of wave glider is very low, so the

\footnotetext{
* Correspondence: fjduan@tju.edu.cn

'State Key Lab of Precision Measuring Technology and Instruments, Tianjin University, Tianjin 300072, China

Full list of author information is available at the end of the article
}

acoustic detection sensitivity of it can be improved considerably. An underwater network lab testbed was described in [4], which contains a real physical environment, such as a set of communication hardware, a programming library, and an emulator.

In the past several decades, the trend of using hydrophone array, rather than single hydrophone, as underwater acoustic information detection method is becoming evident [5-9]. Depending on the detection methods, the ocean acoustic detection can be divided into two kinds: active detection and passive detection. As one typical application of passive acoustic exploration in ocean, the feasibility of short-term seabed earthquake forecasting on East Pacific Rise transform faults was discussed in [5], which is based on the acoustic data acquired passively from six hydrophones emplaced in the eastern Pacific Ocean, Chile. Through research, it is found that it will have a high probability of foreshocks before the main earthquake in some special seabed strata (e.g., in the Eastern Pacific Rise transform faults), compared with very low probability of the aftershocks earthquake. This feature provides specific short-term earthquake prediction possibility of underwater acoustic array data records, which can predict earthquakes above $5.49^{\circ}$ by foreshocks information [5].

As a type of active acoustic detection, underwater seismic exploration is one of the main technical means to implement the marine acoustic exploration of potential seabed oil, gas resources reservoir discovery, and refinement of in- 
fill drilling monitor. For instance, four time-lapse seismic measurements in Gullfaks oil field were fulfilled in 1985 (baseline data), 1995, 1996, and 1999. Through time-lapse seismic data, the movement of water injected was analyzed for forecasting recovery factor of the oil field. As a result, the resources recovery can be increased [6]. In the monitoring of oil fields which have been mined, seismic exploration also can be used. By comparing the seismic data of drilling platforms in before mining and mining with the ongoing oil exploration, the difference of sound response between water and the hydrocarbon compound can be used for resources monitoring [7]. Active acoustic detection methods can also be used for real-time monitoring of marine fish density and behavior [8-10]. Compared with the traditional way, the method of ocean acoustic waveguide and hydrophone linear array can implement thousands of square kilometers of real-time imaging, and continuous monitoring in specified sea water. Acoustic data also can be used for monitoring carbon fixation in the deep ocean. By analyzing the data of 1994, 1999, and 2001 in the same seismic reflection exploration region, it can clearly draw the conclusion that data reflected $\mathrm{CO}_{2}$ changes [11].

A distributed data acquisition system for large-scale land-based seismic data acquisition, which called $\mathrm{rDAQ}$, was reported in [12]. Its data transmission medium is Gigabit Ethernet, file storage format is SEG-D. Clock synchronization of multiple data acquisition node in chain system is useful to improve the performance of hydrophone array. In [13], a new type of synchronous correction method was discussed, which pass through the master-slave clock recovery system to achieve multiple ADC synchronization of data acquisition in distributed data acquisition nodes. The system described in [13] is the basis of this article.

In addition, the hydrophone arrays can also be used for marine seismic exploration underwater acoustic communications and other fields $[14,15]$.

This article presented a type of hydrophone linear array which can be used in the acoustic explorations in shallow water. Its high-precision sampling clock synchronization mechanism was illustrated detailed, as well as real-time data storage method. In the end of the article, we implement a field data collection experiment in Qilihai reservoir in the suburban of Tianjin. The data results show that the system is stable, and can be used for acoustic detection of shallow waters.

\section{Composition of the hydrophone linear array}

Depending on the different location, hydrophone linear array can be divided into on-board equipment and underwater equipment. On-board equipment mainly includes host computer, on-board interface node and power supply, and so on. Underwater equipment is the main body of the system, includes hydrophone groups, which are uniform distribution of linear type; acquisition nodes, which are used to digitize and transmit underwater acoustic signal; head node, which dedicate to communicate with the onboard equipment; and so on. Every data acquisition node includes Data Acquisition Unit (DAU), Data Transmission Unit (DTU), Synchronous-clock Transmission Unit, and Commands Transmission Unit. Every DAU processes 16 channels acoustic signals by 24-bits analog-to-digital converter. Meanwhile, DTU is used to transmit all the data acquired by cascade-type communication channel.

As illustrated in Figure 1, the working procedures of the hydrophone array are as follows: hydrophones convert underwater sound waves into electrical signals to the DAU in the local acquisition node. After different lengths of unshielded twisted pair transmission, electrical signal is amplified and preprocessed by conditioning circuits before evenly distributed multi-channel $\Sigma-\Delta$ ADCs (Sigma-Delta Analog Digital Converter, ADC), which sampled signal 4000 times per second. The sigma-delta architecture is the widely used converting method in the modern high-resolution ADC. The procedure of sigma-delta includes two sections, delta modulation and digital filter. In delta modulation, the analog signal is quantized by a one- or multi-bits ADC. Then, the output is feedback and converted to an analog signal with a DAC. After that, the signal subtracted from the input after passing through an integrator [16]. Sampling data pass through the local asynchronous serial communication thoroughfare to the DTU. Data are packaged into a transmission frame and then transmitted to the head node through a cascade-type data channel.

Head node itself does not have the responsibility of acoustic information acquisition. Mostly, its switching function includes data stream and commands frame. Meanwhile, its duty also involves unified sampling synchronous clock generation and output. The information transmission medium between Head node and Host Computer Interface Node (HCIN) is single mode optical fibers. HCIN receives the data uploaded by head node. Owing to the large amount of data generated through uninterrupted work for a long time, host computer uses the PCI interface as data receiving interface. Data in host computer are stored in real time by the SEG-Y file format. Transmission direction of the command signal is contrary to data stream: host computer -> USIN -> optic fiber $->$ head node $->$ acquisition node (Figure 2).

\subsection{Sampling clock synchronization}

As shown in Figure 3, the clock synchronous system can be divided into clock source (master clock) and lots of data acquisition nodes (slave clock).

Each node interval cascade arranged about $18 \mathrm{~m}$ away. The output frequency of the TCXO is $16.384 \mathrm{MHz}$ which is called $f_{h}$. Transmission clock $f_{1} \sim f_{\mathrm{ml}}$ and each 


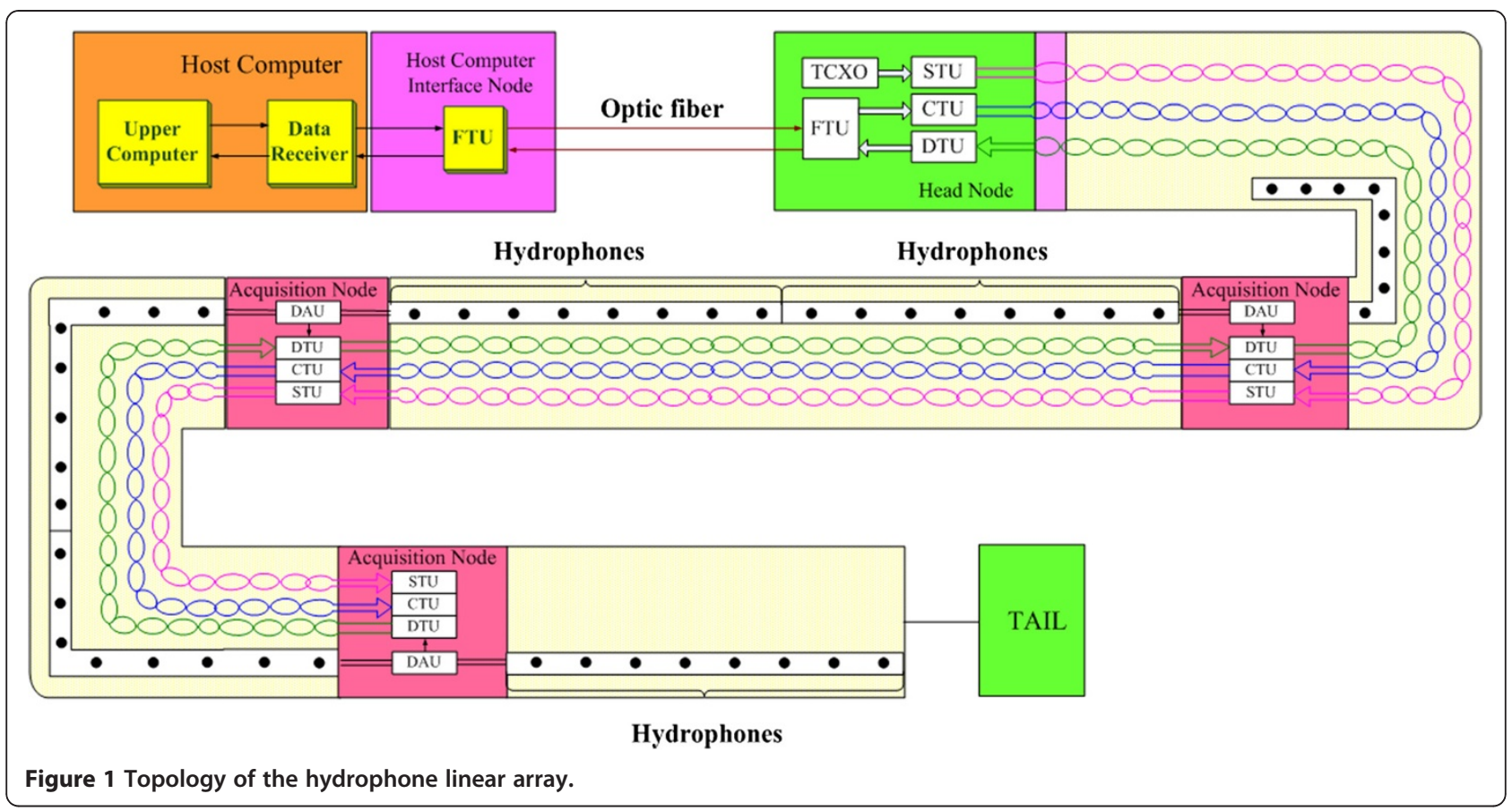

node sampling data output pulse frequency of $f_{1 \mathrm{~d}} \sim f_{\mathrm{md}}$ was $4 \mathrm{kHz}$. As previously mentioned, the synchronization error of data output ticks in all acquisition nodes (i.e., $f_{1 \mathrm{~d}} \sim f_{\text {md }}$ phase error) should as small as possible. Because the array transmission channel did not support the whole Ethernet protocol, thus high-speed clock $f_{h}$ or sync message cannot be transmitted directly in the channel. Instead, the frequency should be divided on $f_{1}$ before transmission. Meanwhile, the high edge steepness and time precision of the waveform still need to be keeping. Each slave clock in acquisition nodes is generated by frequency-doubling from the transmitted clock through a phase-locked loop (PLL). Two inputs of the comparator for PLL error are, respectively, connected with $f_{1} \sim f_{\mathrm{ml}}$ and $f_{1 \mathrm{~d}} \sim f_{\mathrm{md}}$. The purpose of phase locking is eliminating the phase difference of two clock signal inputting the comparator. The PLL output is restored from the high-speed sampling clock $f_{1 \mathrm{~h}} \sim f_{\mathrm{mh}}$.

When the electrical signal transmitted in a unit length of the conductors or printed circuit traces, it is proved

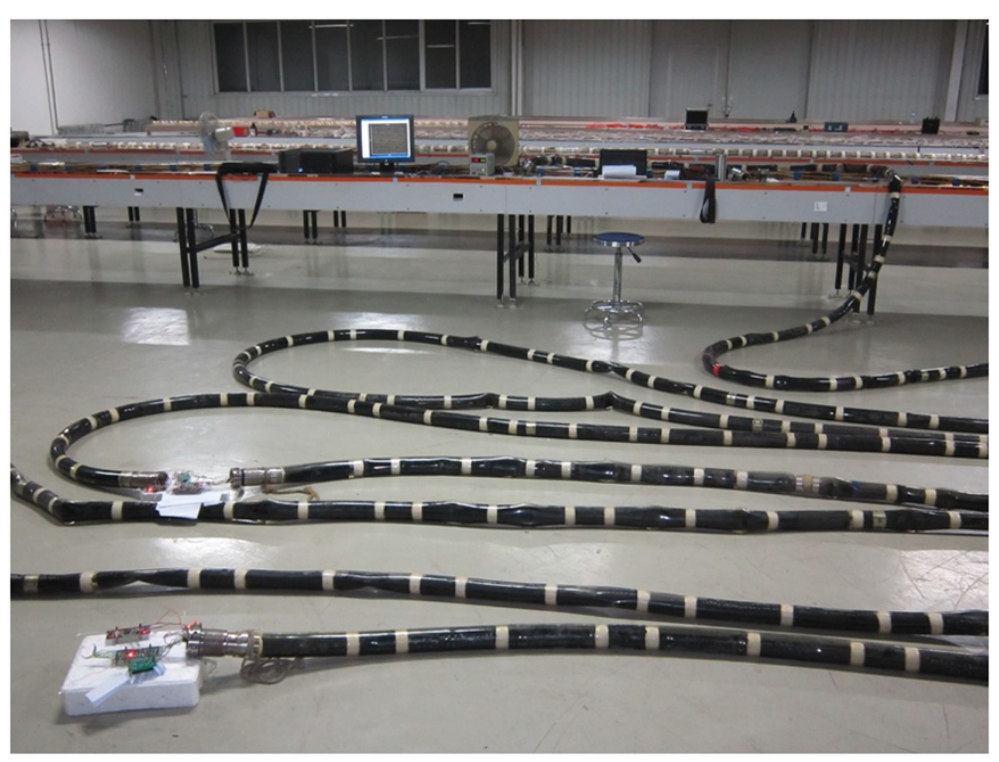

Figure 2 Commissioning of the hydrophone linear array before field testing. 


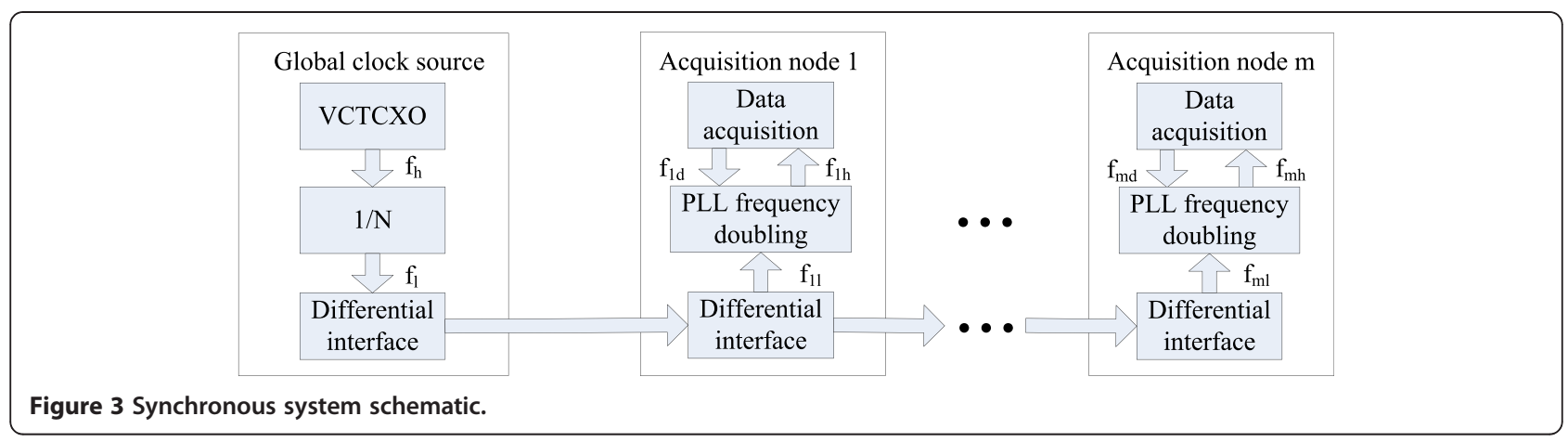

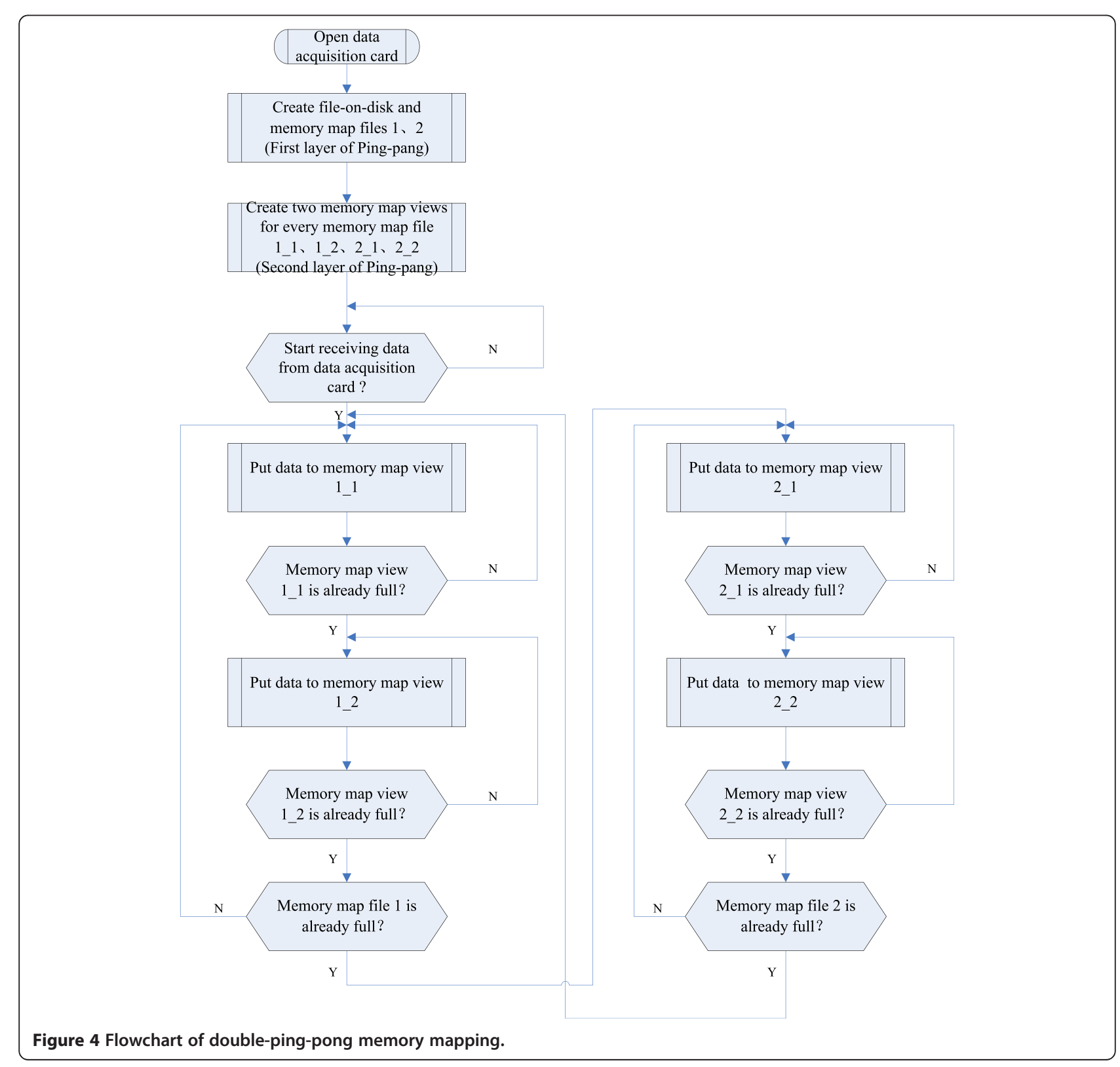




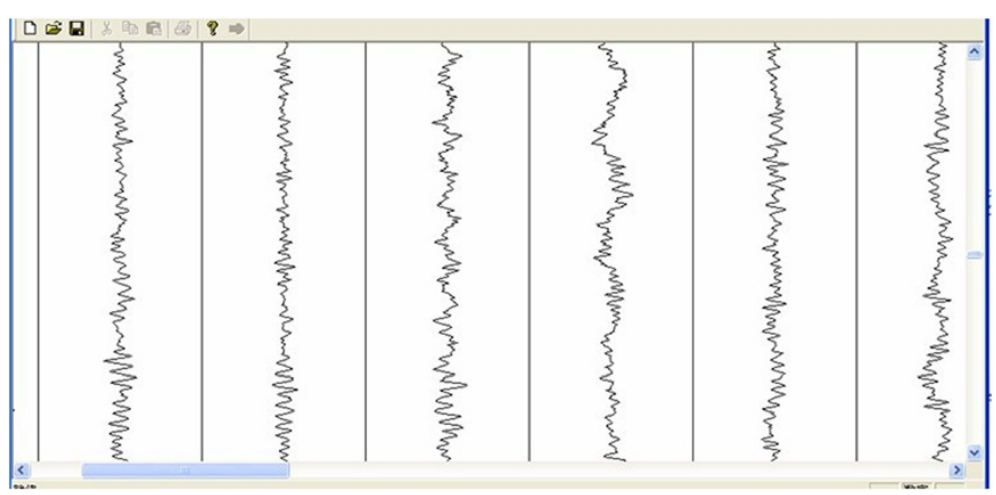

Figure 5 Waveform display graph in host computer.

that there will be a certain time delay between terminal and signal source (called propagation delay unit, $t_{\mathrm{p}}$ ), which is proportional to the square root of the dielectric permittivity of the signal channel insulate material. For example, the dielectric constant of air is 1.0 , thus $t_{\mathrm{p}}$ of radio wave is about $3.35 \mathrm{~ns} / \mathrm{m}$. The interval of dielectric constant for outer polyethylene sheath in unshielded twisted wire is between 1.8 and 2.8. Its $t_{\mathrm{p}}$ is between 4.45 and $5.60 \mathrm{~ns} / \mathrm{m}$.

The transmission delay of synchronous clock is shown in formula (1). On the left side of Equation (1), $t_{\mathrm{ns}}$ is the total delay of $n$th acquisition node clock.

$$
t_{n s}=t_{d}+2 \cdot t_{c}+l_{n} \cdot t_{p}+t_{n m}
$$

where $t_{\mathrm{d}}$ is the time delay of frequency divide in the clock source; $t_{\mathrm{c}}$ is the time delay of differential interface; $l_{n} t_{\mathrm{p}}$ is the time delay of transmission delay for $n$th acquisition node; $t_{\mathrm{nm}}$ is the time delay of the PLL frequency multiplication in the $n$th acquisition node.

The distance between clock source and acquisition nodes named $l_{\mathrm{n}}$ increases linearly with the increase of number $n$th in formula (2). All the values of $l_{\mathrm{n}}$ are known exactly, other errors in synchronous system are determined as well. Therefore, it is possible by means of software to compensate for the total delay in the $n$th node clock $t_{\mathrm{ns}}$, to further improve the accuracy of the clock.

\subsection{Real-time data storage}

By using the memory-mapped data files in Windows system, we can map the files on the disk to the address space of the software processes. Before the process can access memory-mapped file data from its own address space, Windows requires a process to obtain a predetermined

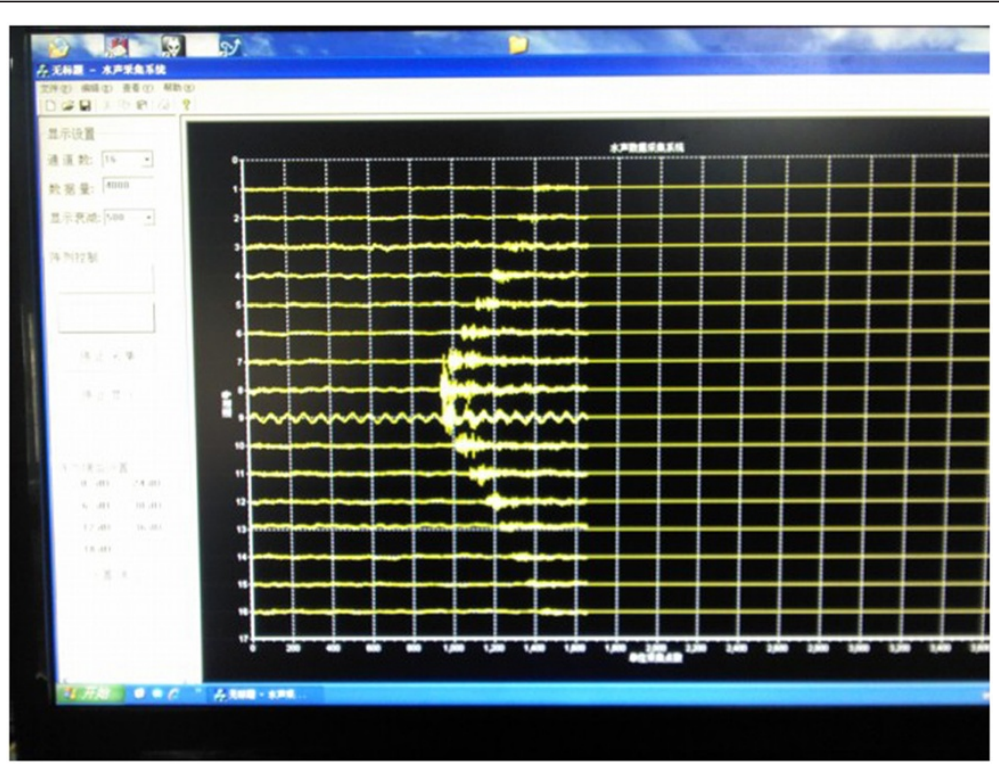

Figure 6 Waveform of the real acoustic data acquisition experiment. 


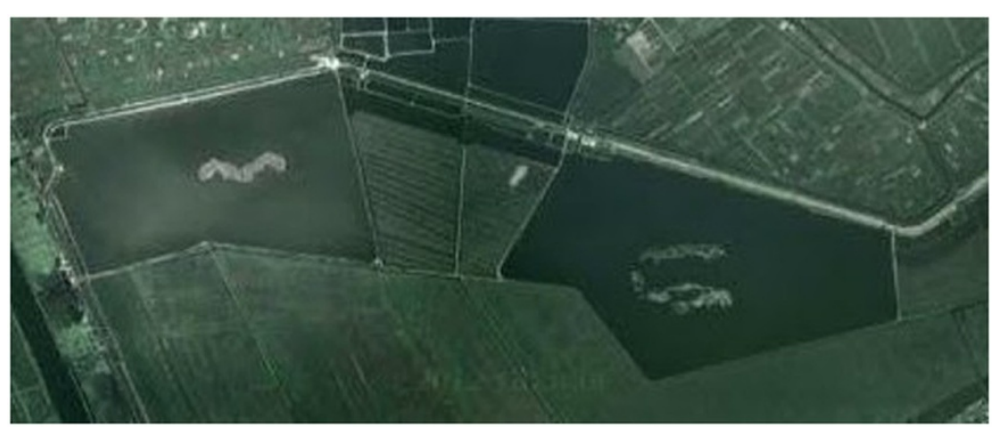

Figure 7 QILIHAI reservoir where the data acquisition experiment carried out.

address space in the mapped view area, and to ensure that the view area is accessible for this process. The view mapped only a small part of the data to a disk file every time. It will re-establish a new view of mapping each time when the view storage is done. The starting address should be increased properly.

The real-time storage of data uses the double-ping-pong memory-mapped file structure. The workflow of data storage was shown below. "File 1" and "file 2" are two memorymapped files. There are four memory-mapped views which named "memory mapping 1_1", "memory mapping 1_2", "memory mapping 2_1", and "memory-mapped 2_2". When the system is running, the host computer establishes two memory-mapped files and four map views. Then host computer receives data triggered by acquisition card interrupt constantly generated from PCI interface. The data are saved to "memory-mapping 1-1" first. When "memorymapping $1-1$ " is full, "memory mapping $1 \_2$ " will continue to save the data immediately. If mapping file "File 1" is full, second mapping file "file 2" will transferred to storage continuously, as shown in Figure 4.

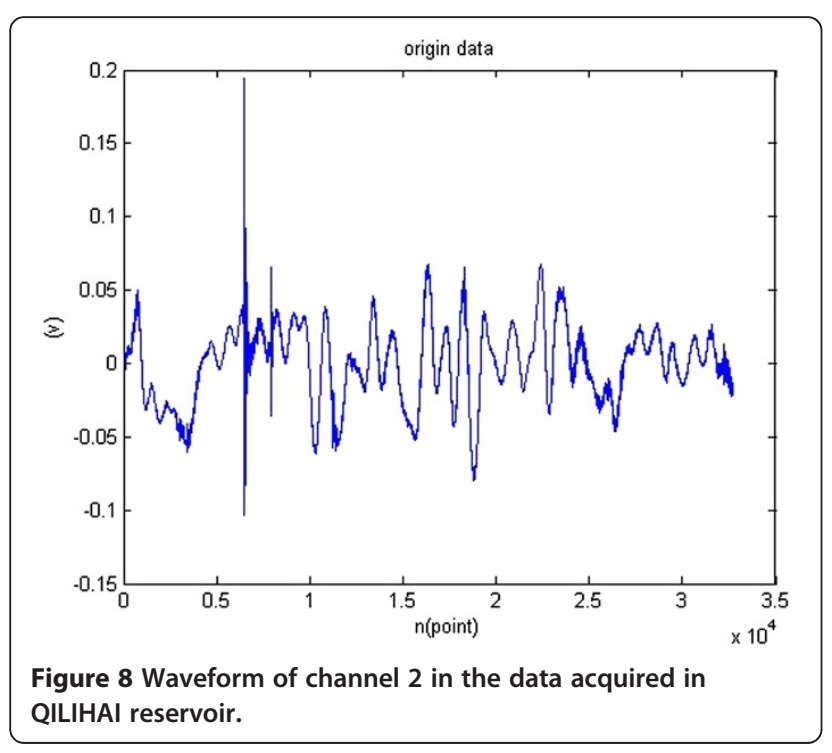

\section{Workbench testing and field data acquisition}

Prototype of hydrophone array was established, which has 24 hydrophones and 2 acquisition nodes. The distance between every hydrophone is $2 \mathrm{~m}$. Figure 5 shows the data acquisition experiment held in the laboratory. The waveform of the display window is a real-time acquisition of the sound information of the hydrophone array. The results show that the hydrophone array system can store the received data realtime, and the echo waveform displayed at fixed time intervals. The acoustic acquisition system software in the host computer achieves reliable storage and waveform display, through memory-mapped access, and double-ping-pong structure. This article has developed a variety of software systems for different applications. Figure 5 shows the main interface window used in laboratory testing. The user interface mainly includes real-time waveform echo display window and the configuration of array parameter window. Real-time data storage module runs in the background. Storage file format is a standard format widely used in various domestic and seismic exploration, named SEG-Y. In the longitudinal direction of the

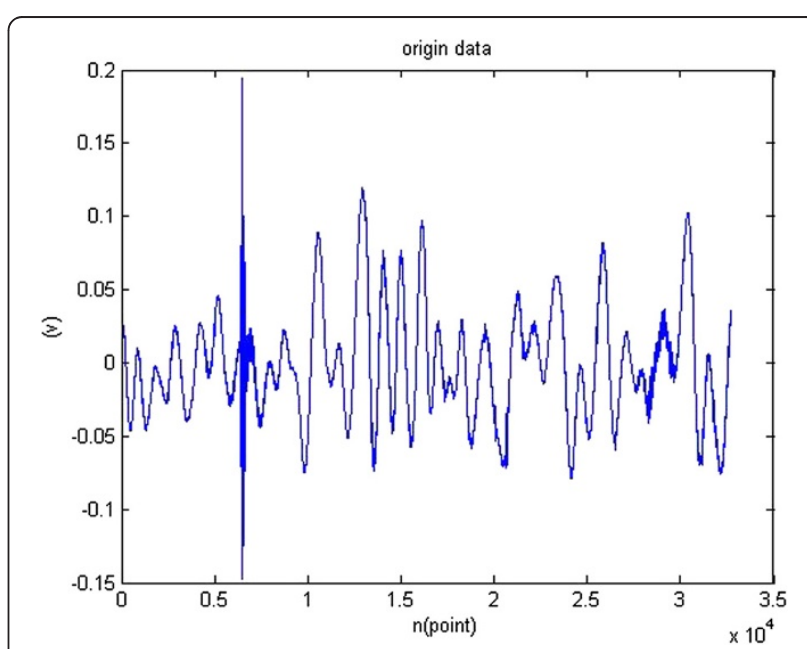

Figure 9 Waveform of channel 3 in the data acquired in QILIHAI reservoir. 
waveform, curves are the real-time acoustic waveform of hydrophone in time domain. Real-time echo display through the software platform can achieve real-time storage of various underwater acoustic waveform of signal.

The actual experiment was held in October 2012. Figure 6 shows the real echo waveform in the experiment nearby a point sound point placed in the middle of the array. Its wavefront propagation path can clearly be seen. So, it can be concluded that the system of data acquisition, transmission, and PC-based data storage are working well.

The data acquisition experiment of hydrophone array was held in autumn in QILIHAI reservoir in Figure 7 which is located in Tianjin, China. The total area of QILIHAI reservoir is about $16.26 \mathrm{~km}^{2}$, average depth is 4 $\mathrm{m}$, so it is rich scattering and reflecting environment. Hydrophone array towed with a ship, arranged linearly closed to the surface of water. Distance from ship to array is $40 \mathrm{~m}$. The sound source is a point-like device which worked on the water-surface. The underwater acoustic transmission abides with the near field model of the spherical wavefront.

Figures 8 and 9 below illustrate the actual underwater acoustic waves of two channels in hydrophone array. It can be seen that synchronization effect in the array is quite well. And sensors are able to respond effectively to the acoustic fluctuation in the water. Moreover, we can see the clutter and noise are noticeable, owing to the little distance from sound source, and shallow environment of the exploration.

\section{Conclusion}

Underwater acoustic measurement has practical significance in the ocean resources exploration, the ecological environment monitoring, and other occasions. Hydrophone array with linear formation is widely used in such fields. With high-precision sampling clock synchronization system, we can obtain more accurate data which contain more information, through the experiment carried on QILIHAI reservoir in Tianjin, China.

Moreover, it is shown that towed linear array can be used in shallow environment with high-speed real-time data storage. The synchronous acquisition method is less influenced by environment, and the data storage system can accurately implement the mass data storage.

\section{Competing interests}

The authors declare that they have no competing interests.

\section{Acknowledgment}

This study was supported by the grants Program for New Century Excellent Talents in University (NCET), TOA (KX2010-0006), and TSTC (11ZCKFGX03600) in China.

\section{Author details}

'State Key Lab of Precision Measuring Technology and Instruments, Tianjin University, Tianjin 300072, China. ${ }^{2}$ College of Physics \& Electronic Information, Tianjin Normal University, Tianjin 300387, China.
Received: 11 December 2012 Accepted: 21 January 2013

Published: 19 February 2013

\section{References}

1. CR Tunnicliffe, R Barnes, X Dewey, Major advances in cabled ocean observatories (VENUS and NEPTUNE Canada) in coastal and deep sea settings, in US/EU-Baltic International Symposium (IEEE/OES, 2008), pp. 1-7

2. J Heidemann, M Stojanovic, M Zorzi, Underwater sensor networks: applications, advances, and challenges. Philos. Trans. R. Soc. A 370, 158-175 (2012)

3. B Bingham, N Kraus, B Howe, L Freitag, K Ball, P Koski, E Gallimore, Passive and active acoustics using an autonomous wave glider. J. Field Robot. 29(6), 911-923 (2012)

4. Z Z Peng, J Cui, B Wang, K Ball, L Freitag, An underwater network testbed: design, implementation and measurement, in Proceedings of the Second Workshop on Underwater Networks (ACM, 2007), pp. 65-72

5. JJ McGuire, MS Boettcher, TH Jordan, Foreshock sequences and short-term earthquake predictability on East Pacific Rise transform faults. Nature 434, 457-461 (2005)

6. M Landrø, LK Strønen, P Digranes, OA Solheim, E Hilde, Time-lapse seismic as a complementary tool for in-fill drilling. J. Petrol. Sci. Eng. 31, 81-92 (2001)

7. AM Swanston, PB Flemings, JT Comisky, DP Best, Time-lapse imaging at Bullwinkle Field, Green Canyon 65, offshore Gulf of Mexico. Geophysics 68 (5), 1470-1484 (2003)

8. NC Makris, P Ratilal, DT Symonds, S Jagannathan, SW Lee, RW Nero, Fish population and behavior revealed by instantaneous continental shelf-scale imaging. Science. 311, 660-663 (2006)

9. NC Makris, P Ratilal, S Jagannathan, Z Gong, M Andrews, I Bertsatos, OR Godø, RW Nero, JM Jech, Critical population density triggers rapid formation of vast oceanic fish shoals. Science 323, 1734-1737 (2009)

10. NC Makris, S Jagannathan, A Ignisca, Ocean acoustic waveguide remote sensing: visualizing life around seamounts. Oceanography 23(1), 204-205 (2010)

11. R Arts, O Eiken, A Chadwick, P Zweigel, L van der Meer, B Zinszner, Monitoring of $\mathrm{CO} 2$ injected at Sleipner using time-lapse seismic data. Energy 29, 1383-1392 (2004)

12. $\mathrm{P}$ Cao, $\mathrm{S}$ K-z, Y J-f, R F-m, Design of a large remote seismic exploration data acquisition system, with the architecture of a distributed storage area network. J. Geophys. Eng 8, 27-34 (2011)

13. J J-j, DUAN F-j, J Chen, C Zhang, K Wang, C Z-j, Calibration method of the time synchronization error of many data acquisition nodes in the chained system. J. Geophys. Eng 9, 413-422 (2012)

14. M Stojanovic, Recent advances in high-speed underwater acoustic communications. IEEE J. Ocean. Eng. 21(2), 125-136 (1996)

15. AO Bicen, $A B$ Sahin, $O B$ Akan, Spectrum-aware underwater networks: cognitive acoustic communications. IEEE Veh. Technol. Mag. 7(2), 34-40 (2012)

16. W Kester, ADC Architectures III: Sigma-Delta ADC Basics, 2009. http://www.analog.com/static/imported-files/tutorials/MT-022.pdf

doi:10.1186/1687-1499-2013-35

Cite this article as: Chen et al:: Offshore towed hydrophone linear array: principle, application, and data acquisition results. EURASIP Journal on Wireless Communications and Networking 2013 2013:35.

\section{Submit your manuscript to a SpringerOpen ${ }^{\circ}$ journal and benefit from:}

- Convenient online submission

- Rigorous peer review

- Immediate publication on acceptance

- Open access: articles freely available online

- High visibility within the field

- Retaining the copyright to your article

Submit your next manuscript at $>$ springeropen.com 